\title{
Dual-nature biometric recognition epitome
}

\section{Shahzad Ashraf* and Tauqeer Ahmed}

College of Internet of Things Engineering, Hohai University Changzhou China

Received: 05 June, 2020

Accepted: 22 June, 2020

Published: 23 June, 2020

*Corresponding author: Shahzad Ashraf, College of Internet of Things Engineering, Hohai University Changzhou China, E-mail: nfc.iet@hotmail.com; shahzadashraf@hhu.edu.cn

Keywords: Multi-biometric; Appellative; Shrewd; Sagacious; Face mask

https://www.peertechz.com

r - - - - - - - - - - - - - -

Check for updates

Check for updates

\section{Abstract}

All humans are born with unique physically identified body characteristics to other persons which remains unchanged throughout life. These characteristics are taken into account by the emerging technology to get recognized from person to person. The technology used by the traditional human identification system sometimes becomes inefficient when data or images received are not up to the acceptable quality mark or when a person has a face covered with mask-like during epidemic virus fistula. In order to overcome such human recognition challenges, a Multi-biometric sustainable approach (D-nb) has been proposed using two uniquely identified modalities like foot and iris. This approach shrewdly identifies and makes the bodacious recognition among humans and suggests the sagacious result which is foremost better than the traditional biometric system.

\section{Introduction}

Today a biometric recognition process is very popular from both physiological and behavioral points of view for identification and authentication of individuals. If both are the same as the decision, the test picture corresponds to the store picture and it is checked that it is a mapping operation [1]. In order to identify the test image with the saved database, the person is identified or does not decide whether it is only one to many mapping systems. Some prominent biometric identification features are being used as [2].

Face biometric: Face recognition is a technique in which a person is automatic identified and verified using digital image [3].

Ear biometrics: Ear biometrics uses features or characteristics of ear for matching. This is a stable and not changes with age [4].

Fingerprint biometrics: A fingerprint is one of the most popular biometrics used in last few decade for recognition of a person. Ink is used for taking an impression of finger and thumb images on paper [5].

Hand Geometry biometrics: Now palm print is also used as a biometric because each person has no changes in lines of palm print during the growth [6].
Voice biometrics: Voice biometric comes under the behavioral characteristics each person has different sound, pitch and speaking style [7].

Signature biometrics: The making of signature. Signature are measured as writing style of a person that means holding the pen, pressure on paper and the direction of letter is different each parson has its own style [8].

It accomplishes in two steps; one is the training process and the other is the testing process.

\section{Training process}

o Input image is captured or acquired by camera.

o An image is pre-processed means image resizing, reformatting.

o Feature extraction is performed.

o Prepared database.

Testing of image: It is close to the preparation phase, but balanced. The processes of training and testing are described in Figure 1

There are numerous challenges faced by the Uni-biometric system such as: 


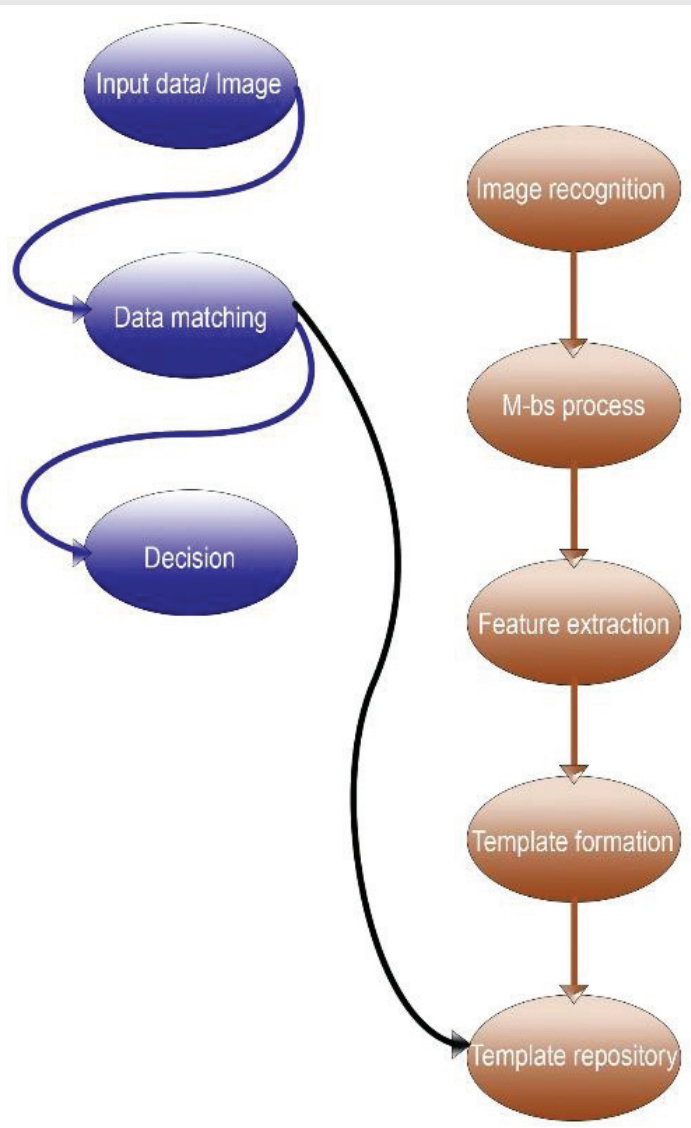

Figure 1: Information training flow process.

- $\quad$ Noise

- Intra-class variations

- Non-universality

- $\quad$ Spoof attacks

Multi-biometrics offer opportunity to take more than two biometric features, to combine them to overcome unimodal biometric system limitations and to achieve optimal results [9]. We've been using two biometric foot and iris for a multibiometric device. Sprints have been developed, iris are special and not changed during a person's age as a reflective layer structure.

A critical comparative analysis of foot and iris modalities with other available practices have been epitome in Table 1.

The rest of the article is arranged as, Related work is addressed in section II, section III presents the proposed methodology, section IV demonstrates the performance obtained through results while concluding remarks are given in section $\mathrm{V}$.

\section{Related works}

Many researchers have made significant contributions reported [10], that the biometrics is becoming more commonly used in several places through different biometric device such as bank, institute, airports. Multi-biometric is preferred in authentication-based image processing applications. The authors [11], introduced a multi modal systems working on physiological and demography information for identification of person.

The author [12], links open overlay panel introduced a biometric system for person recognition that shown the improvement accuracy of the recognition system. Another research work on face and ear-based hybrid system has been presented by [13]. This system is used Iterated Function concept in which images are compression and indexed. Sarfraz, et al. [14], designed a multi-biometric system that combines face modalities and foot modalities using PCA [15], classifier for face and wavelet transformer for foot and concatenated after normalization process to obtain a matching score and take a decision. Another author Davrondzhon [16], proposed a system for multimodalities biometric that combines face, ear and iris using PCA, Eigen image [17] and hamming travers [18], classifier for feature extraction, sum rule method is used for fusion to calculate matching score. They introduced recognizing person utilizing multiple biometric traits and their advantages such as high accuracy and robustness, that increased recognition performance. Herbadji, et al. [19], developed a Multi-biometric system, which combined face and foot modalities at decision level using PCA classifier for face and wavelet transformer for foot and concatenated after normalization process to obtain matching score [20].

\section{Methodology}

The body parts can be paired with the other body elements in several potential ways, leading to very diverse machine architectures. Particularly in parallel or in cascade, the Iris footprint can be combined and therefore further findings are arranged as:

\section{Iris biometrics}

Iris recognition work on the colored part of eye that is isometrics surrounds by pupil known as iris. Iris image is captured using high quality camera every person has a unique iris. Process of calculating patterns are very complex it stored in bit format as a template illustrated in Figure 2. Hamming traverse method is used for calculating the distance between test image and stored template if the distance is zero that indicate the test image and training image is same otherwise, we can say both images are different.

\section{Footprint biometrics}

Identification of peoples by fingerprint is becoming a wonderful success. Measurement such features are not a complicated process some transformation such as furrier, haar are available [21]. Each parson has completely different footprint and it is easy to capturing without any special requirement both leg images can be used for recognition [22]. Captured foot image needs some additional approach such as cropping and resizing. Figure 3 displays the picture captured by high quality camera than the RGB image converted into gray scale after that resize them for preparing the database. 
Table 1: Critical comparative analysis of biometric authentications with proposed technique.

\begin{tabular}{|c|c|c|}
\hline $\begin{array}{l}\text { Recognition } \\
\text { method }\end{array}$ & Flexibility & Challenges \\
\hline Face & $\begin{array}{l}\text { Most favorable, works foremost } \\
\text { better while identifying masses } \\
\text { of people who spent just } \\
\text { seconds before a camera or } \\
\text { scanner }\end{array}$ & $\begin{array}{l}\text { Age invariant, thermal imaging due } \\
\text { to multi-feature extractions from } \\
\text { small and large database, single face } \\
\text { identification with occlusion, Facial } \\
\text { expressions like (joy, sadness, anger, } \\
\text { fear, disgust and surprise), poses }\end{array}$ \\
\hline Fingerprint & $\begin{array}{l}\text { Most common and low cost, } \\
\text { no two fingerprints were } \\
\text { ever found to be the same in } \\
\text { over } 140 \text { years of worldwide } \\
\text { fingerprint comparison }\end{array}$ & $\begin{array}{l}\text { Misrepresentations due to finger } \\
\text { injuries and finger cuts. physical } \\
\text { distortion. }\end{array}$ \\
\hline Hand Geometry & $\begin{array}{l}\text { Can be useful in harsh } \\
\text { environments, do not require } \\
\text { clean conditions, requires very } \\
\text { small database, environmental } \\
\text { factors, such as dry weather } \\
\text { causing drying of the skin, are } \\
\text { not a problem }\end{array}$ & $\begin{array}{l}\text { The hand size restricts the number of } \\
\text { applications. At least two out of } 100 \\
\text { randomly selected persons have the } \\
\text { same hand geometry. }\end{array}$ \\
\hline Retina scan & $\begin{array}{l}\text { Requires very small file size, } \\
\text { can take place in less than two } \\
\text { seconds }\end{array}$ & $\begin{array}{l}\text { May be a health risk, Requires the } \\
\text { highest level of end-user cooperation } \\
\text { and motivation }\end{array}$ \\
\hline Voice & $\begin{array}{l}\text { Non-intrusive. High social } \\
\text { acceptability, provides quick } \\
\text { and efficient authentication, } \\
\text { natural } \\
\text { input and does not demand any } \\
\text { training }\end{array}$ & $\begin{array}{l}\text { Having low accuracy, an illness like } \\
\text { cold will alter a person's speech, } \\
\text { rendering it difficult or impossible to } \\
\text { recognize absolute }\end{array}$ \\
\hline & & \\
\hline
\end{tabular}

\section{Working ground}

Analyzes the particular characteristics common to all faces such as the distance between the eyes, width of the nose, position of cheekbones, jaw line and chin etc.

The pattern of ridges and furrows on the fingertips, as well as the minutiae points of a specimen print with a database of prints on file.

Based on the geometric shape of a hand-palm size, finger length and width, knuckle distance. Use in industrial environment

It captures blood vessel pattern on the back of the eye, a standard in military and government installations. Spectrogram takes the voice sample then paired with stored voice data
Comparison with proposed technique (Iris and foot)

Iris scanner uses safe and low energy visible infrared light and can recognize at least from 40 feet away. Iris scanners collect approximately 240 biometric features. Throughout one's life, iris patterns remain stable. It is protected by the mechanism of the body itself. For a footprint, the footprint pressure distribution is calculated by means of a pressure sensor pad. Footprintbased authentication is adequately reliable to focus on sensitive data provided by high-security applications without any downside.
Uses checksum mechanism, Cyclic Redundancy Check and private and public key authentication my take longer, Sign issues differ from one cause to the next

\section{Hamming distance method for iris}

Hamming distance approach [23], is applied for a matching iris templates incorporate noise masking also. The distance between two iris templates is calculated in bits format and distance calculated between two iris templates if the distance is minimum that indicate the iris are same otherwise iris are not matched which confirms hamming distance is a reliable and accurate for iris recognition.

The iris recognition process completed in three stages

a. Segmentation: Located the iris region

b. Normalization: Created a consistency of data iris region

c. Feature encoding: Produced templates as set of discriminated features of the iris.

\section{Sequential modified Haar transform technique}

The sequential modified haar wavelet [24], is based on the mapping technique in which integer-valued signals are mapped using the reconstruction of image technique. Wavelet coefficients represented by decimal numbers and each decimal value needed eight bytes for storing the haar value.

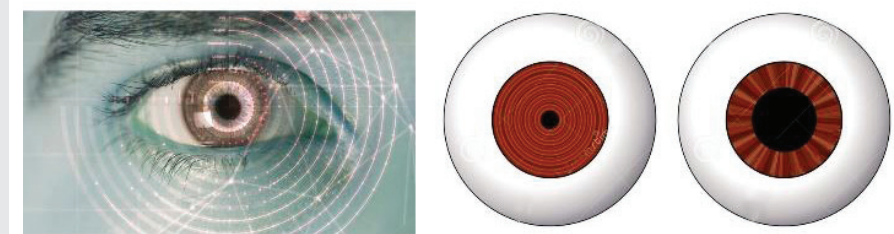

Figure 2: Iris selection initialization process.
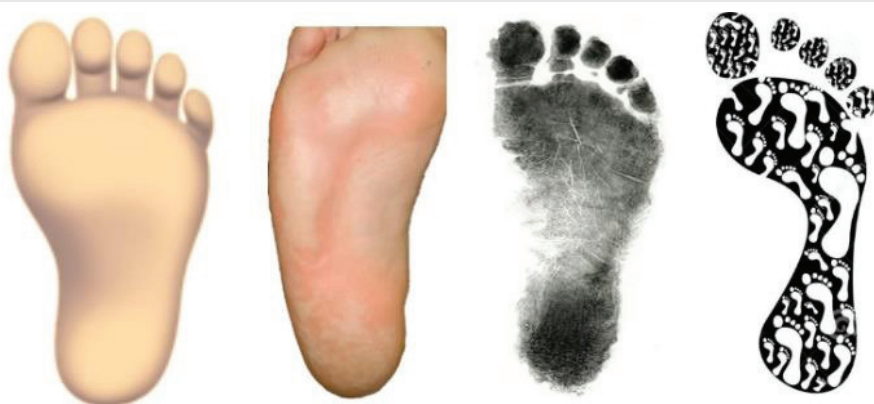

Figure 3: Foot selection initialized stages.

\section{Multi-biometic approach}

The sample images of eye are being consider as input to the system and thereby iris templates are generated as 
output. The discrete bits formation of the image has been obtained illustrated in Figure 4, showing segmentation and normalization stages [25].

\section{Image segmentation}

The control area on the 3-D floor is exposed to the pressure distribution of the person on the medium [26]. Because human physical presence defines the significant pressure surfaces, personality identification can be thus understood through the classification of high pressure surfaces and examination of their formal features [27]. When the scene footprint is compared to other templates, the public protection program can assess or reject the accused individual. The visible footprint arc travels away from the curved surface position and direction which has little to do with the curved surface parameter.

\section{Image noise reduction}

For identification of footprint patterns, edge detection is necessary. For the sake of the gradient of light intensity of a gray-scaled picture it is very difficult to distinguish corners via a Laplacian transformation [28]. Then for edge detection the entire picture must be binarized. To binarize the entire image, a certain threshold value is not adequate. The entire picture is then separated into several sections, and threshold values are autonomously determined for such a segment.

The proposed technique produces $n$ number of iris codes out of $n$ number of eyes tests performed at various time periods from the same person. Using majority voting scheme [29], a unique iris code $x$ is built from the $n$ number of iris code. The suggested framework operates on $x$ and generates codewords, known as Error Corrected Iris Code (ECIC) [30]. These ECICs are composed of iris coding and parity regulation $p$. The hash $h$ is also created from code $x$. Eventually, parity regulates $p$ of the ECIC, matrix $h$ of parity and hash-h render code that are embedded in digital images by transformation of the integrated wavelet and incorporation processing threshold illustrated in Figure 5.

The new and unique feature of the multi-biometric is the speed and precise segmentation of irises, the iris image being captured to reduce identifying errors, producing a vector with distinguishing texture characteristics and a proper dimension to improve the detection and computational efficiency [31]. The sagacious edge detection and circular Hough transforms are used for the segmentation process. The segmented iris is normalized and phase data is extracted by applying LogGrabor filter [32] and encoded shrewdly to produce the refined vector feature through phase quantization method [33]. The iris from real eye object has been extracted after undergoing segmentation, normalization and quantization process as shown in Figure 6 . Unless the iris area has been effectively segmented from the eye object, the next step is to turn the iris region in such a manner that it has set measurements to enable comparisons [34]. Dimensional incoherence [35], between eye images are mainly because of the iris stretching caused by the dilation of pupils from different luminance levels. Other sources of inconsistency include varying imaging distance,

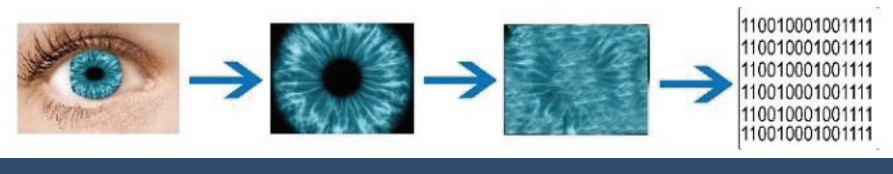

Figure 4: Segmented and Normalization discrete bit formation.

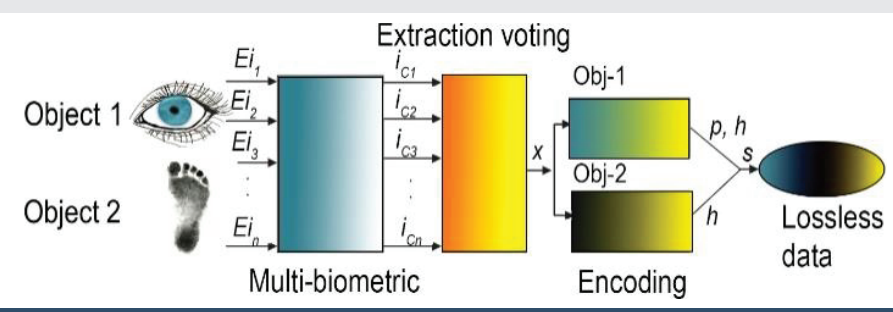

Figure 5: Proposed multi-biometric object processing mechanism.
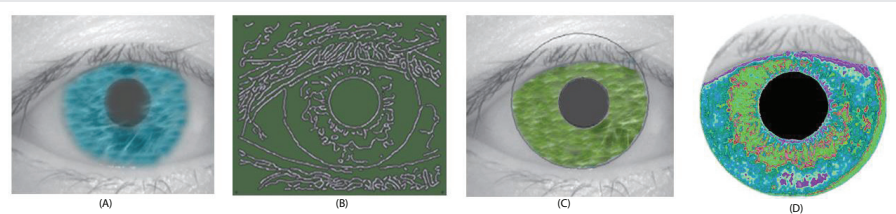

Figure 6: Extracting iris from eye object after segmentation, normalization and quantization processes phases.

(a) The real object.

(b) Sagacious edge detection.

(c) Hough transform results.

(d) multi biometric extracted iris

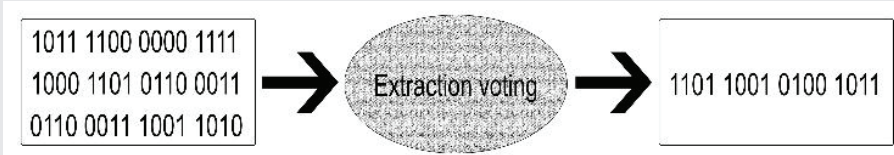

Figure 7: Extracting voting mechanism.

camera rotation, head tilt and eye rotation within the socket of the eye. Construction outcomes of individual iris code $x$ from $n$ number of iris code are achieved using a specific procedure named extraction vote. Figure 7, explains the fabrication of these unique iris $x$ from three sample iris codes. The sparse parity test matrix is used to

describe multi-biometric codes. This small matrix is often generated at random, depending on the sparsity limitations [36]. Referring Figure 5, The valid message bits are processed by the multi-biometric phase when satisfy the condition thereby extraction voting occurs. After creating a specific iris code $x$, each column in iris code $x$ is considered to be a message in multi-biometric encoding and encoded to render ECIC with the aid of generator values. The special iris code $x$ is converted into hash $\mathrm{h}$, often referred to as cancellable iris code. Table 2 shows factual score, imposter score and threshold value for object 1 and 2 respectively. The Iiris and foot biometrics were

tested individually and Table 3 show False Accept Rate (FAR) [4], and False Reject Rate (FRR) [37], of Iris and foot. The weight of all individual iris and foot modalities was calculated, shown in Table 4. Iris recognition and foot recognition algorithms produced dissimilarity scores [38]. 
The Min-Max normalization technique [39], was used to convert all dissimilar data into similar data shown in Table 5.

\section{Iris and foot fusion mechanism}

By adopting weighted sum rule procedure, the matching scores generated for both modalities are fused and combined matching scores are generated as Equation 1.

$$
S=\left(W_{1} * I\right)+\left(W_{2} * F\right)
$$

Considering $S$ as a final score obtained by averaging and then adding the scores of iris (I) and foot (F). If the results match successfully, person will eventually be verified, otherwise, depending on the matching score.

Table 6 shows the weight value of iris and foot after the fusion of two traits.

Matching score was calculated for two modalities using their weight Table 7 shows the matching score for combination of two traits iris and foot.

\section{Performance and results}

For getting performance results, nearabout 100 sampling objects (eye and foot) among 500 images were considered.

Table 2: The factual score, Imposter score and Threshold value for iris and foot images.

\begin{tabular}{|c|c|c|c|}
\hline Traits & Genuine Score & Imposter Score & Threshold Value \\
\hline Iris & $1.7121 \mathrm{E}+04$ & $1.9082 \mathrm{E}+04$ & $1.7330 \mathrm{E}+04$ \\
\hline Foot & $2.2277 \mathrm{E}+04$ & $2.6613 \mathrm{E}+04$ & $2.2325 E+04$ \\
\hline \multicolumn{4}{|c|}{ Table 3: FAR and FRR for iris and foot images. } \\
\hline \multicolumn{2}{|c|}{ Traits } & FAR & FRR \\
\hline \multicolumn{2}{|c|}{ Iris } & $1.1010 \mathrm{E}+00$ & 9.8793E-01 \\
\hline Foot & \multicolumn{2}{|c|}{ 1.1921E+00 } & $9.9785 \mathrm{E}-01$ \\
\hline
\end{tabular}

Table 4: Weight for all Modalities.

\begin{tabular}{|c|c|}
\hline Traits & Weight \\
\hline Iris & 0.89 \\
\hline Foot & 0.83 \\
\hline
\end{tabular}

Table 5: Normalized Score.

\begin{tabular}{|c|c|}
\hline Traits & Normalized Score \\
\hline Iris & 0.05 \\
\hline Foot & 0.07 \\
\hline
\end{tabular}

Table 6: Weight for each Trait in Fusion of two Traits.

\begin{tabular}{|l|l|l|}
\hline Traits & Iris & Foot \\
\hline Iris + Foot & 0.517 & 0.482
\end{tabular}

Table 7: Matching Score for each Trait after fusion.

\begin{tabular}{|c|c|}
\hline Traits & Score \\
\hline Iris + Foot & 0.292 \\
\hline
\end{tabular}

For each iris object, the four samples were taken for multibiometric process. The remainder of the iris representation in the repository is linked to the other iris in the authentication process. A total number of $(500 \times 499) / 2=124,750$ comparisons were made, out of which 345 intra-group matches were found and another 116,602 inter-group matches were recorded. Figure 8 , exhibits a total intra-group and inter-group distribution. It has been observed that travers between intra-group and Intergroup is exorbitant and the portion occurring between the intra-class and the inter-class is quite trivial, thereby nearly $100 \%$ of the right identification levels are obtained. In-order to determine the error correction capacity of D-nb, Reed Solomon (RS) [40], code is being applied which eventually generate the results as illustrated in Figure 9. The selected RS and D-nb curves overlaid on top of the real and impostor normalized HD distributions. RS correction curves are also notably lower than the D-nb curves. In comparison, the RS encoding is often less granular than the D-nb. It leads in efficiency loss, with the False Rejection Rate (FRR) and the False Acceptance Rate (FAR) levels.

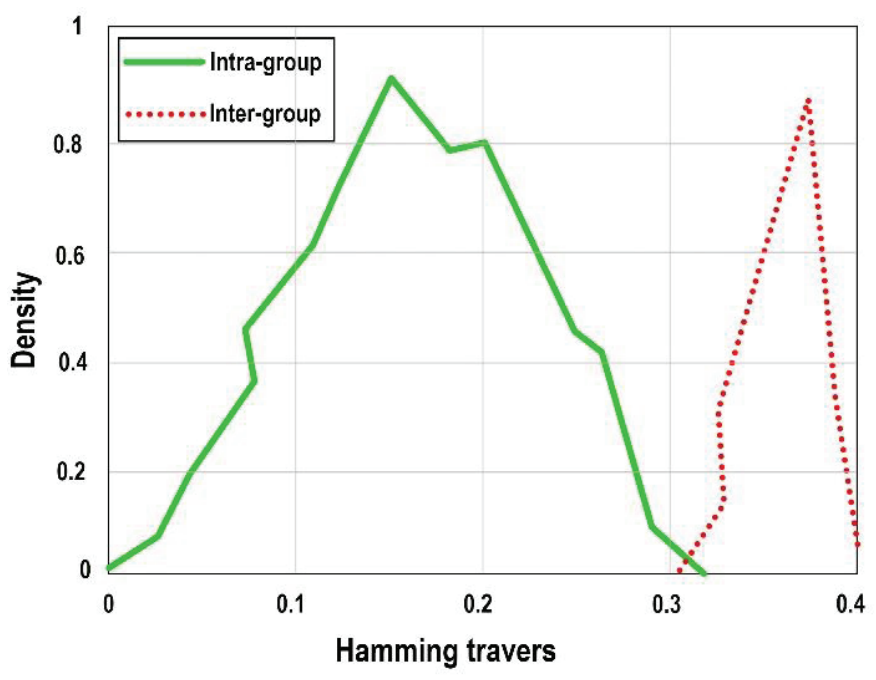

Figure 8: Intra and inter-group travers distribution.

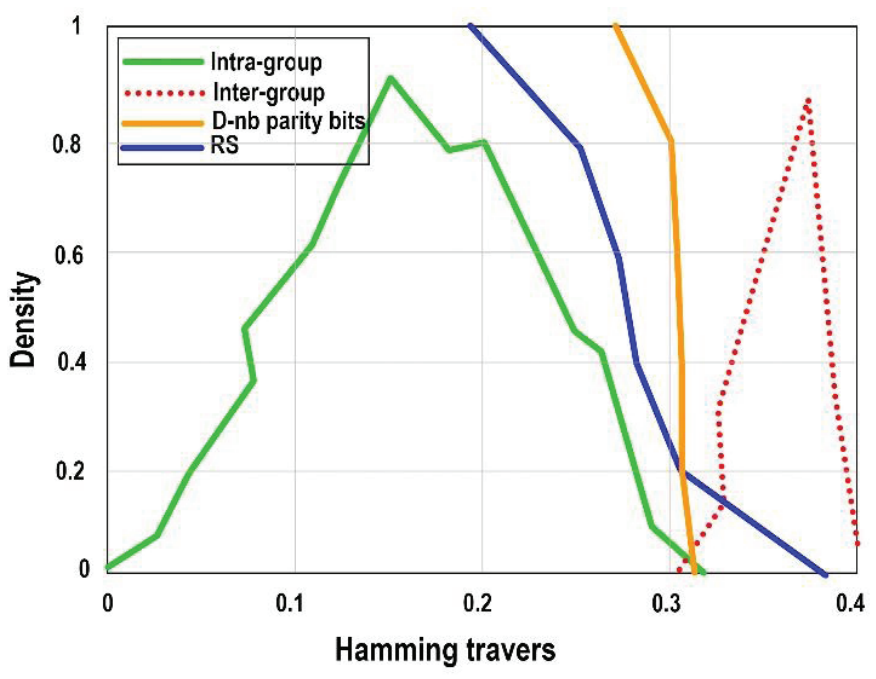

Figure 9: Determination if error correction mechanism. 


\section{Conclusion}

The multi-biometric system employed biometric features of iris and foot. For hamming archways and sequential modified classifier approaches of haar transformation, weight was calculated of each biometric element. After normalization, the knowledge was combined. The Iris is divided into an easy and quick technique, based on the Sagacious edge detector and Hough transformation. For other approaches, the actual FRR and FAR are 2.0 and $0.38 \%$, while the FRR and FAR suggested by $\mathrm{D}-\mathrm{nb}$ are 1.87 and 0.365 percent. Due to the improved steepness and granularity properties, D-nb codes have shown to result in better recognition performance than RS codes. The framework uses D-nb codes for lower FRR and FAR.

As a conclusion, it can be claimed that, compared to other reported technologies, the proposed system cam up with $98 \%$ accurate and shrewd performance in terms of reliability, accuracy and continuity.

\section{References}

1. Biometric Recognition: Challenges and Opportunities (2010) The National Academies Press. Link: https://bit.ly/3hPFzD5

2. Yang W, Hu J, Wang S, Wu Q (2018) Biometrics Based Privacy-Preserving Authentication and Mobile Template Protection. Wireless Communications and Mobile Computing 2018: 7107295. Link: https://bit.ly/2BrHNYA

3. MS University (2018) Unlocking new possibilities for biometrics. MSUToday. Link: https://bit.ly/314PH4X

4. False Acceptance Rate and False Recognition Rate in biomemetrics. Link: https://bit.ly/2Ymy7rc

5. Gunasekaran K, Raja J, Pitchai R (2019) Deep multimodal biometric recognition using contourlet derivative weighted rank fusion with human face, fingerprint and iris images. Automatika 60: 253-265. Link: https://bit.ly/3hSmNL9

6. Saini RK, Rana N (2014) Comparison of Various Biometric Methods. Link: https://bit.ly/3fLXGb5

7. Uhl A, Wild P (2007) Personal identification using Eigenfeet, Ballprint and Foot geometry biometrics. In 2007 First IEEE International Conference on Biometrics: Theory, Applications, and Systems 1-6. Link: https://bit.ly/37QWXmd

8. Campisi P, Maiorana E, Neri A (2011) Signature Biometrics. In Encyclopedia of Cryptography and Security, H. C. A. van Tilborg and S. Jajodia, Eds. Boston, MA: Springer US 1208-1210.

9. Ashraf S, Saleem S, Chohan AH, Aslam Z, Raza A (2020) Challenging strategic trends in green supply chain management. Int J Res Eng Appl Sci Jreas 5: 7174. Link: https://bit.ly/3dNy9gn

10. Pandya J (2019) Hacking Our Identity: The Emerging Threats From Biometric Technology. Forbes. Link: https://bit.ly/3hT6xcQ

11. (2020) Multimodal System - an overview. Link: https://bit.ly/3hPRX66

12. Giannakos MN, Sharma K, Pappas IO, Kostakos V, Velloso E (2019) Multimodal data as a means to understand the learning experience. Int $\mathrm{J}$ Inf Manag 48: 108-119. Link: https://bit.ly/2Nje3jh

13. Ashraf S, Gao M, Chen Z, Kamran S, Raza Z (2017) Efficient Node Monitoring Mechanism in WSN using Contikimac Protocol. Int J Adv Comput Sci Appl 8. Link: https://bit.ly/3fGopG2

14. Sarfraz N (2019) Adermatoglyphia: Barriers to Biometric Identification and the Need for a Standardized Alternative. Cureus 11: e4040. Link: https://bit.ly/31752Ca

15. Luaibi MK, Mohammed FG (2019) Facial Recognition Based on DWT - HOG - PCA Features with MLP Classifier. J Southwest Jiaotong Univ 54. Link: https://bit.ly/2CyySFv

16. Gafurov D (2008) Emerging Biometric Modalities: Challenges and Opportunities. 122: 29-38. Link: https://bit.ly/2Ylglzl

17. The essence of eigenvalues and eigenvectors in Machine Learning. Link: https://bit.ly/3dkwKOJ

18. Doriguello JF, Montanaro A (2019) Quantum sketching protocols for Hamming distance and beyond. Phys Rev A 99. Link: https://bit.ly/2AWu6ko

19. Herbadji A, Akhtar Z, Siddique K, Guermat N, Ziet L, et al. (2020) Combining Multiple Biometric Traits Using Asymmetric Aggregation Operators for Improved Person Recognition. Symmetry 12: 444. Link: https://bit.ly/2NwE8f3

20. Ashraf S, Arfeen ZA, Khan MA, Ahmed T (2020) SLM-OJ: Surrogate Learning Mechanism during Outbreak Juncture. Int J Mod Trends Sci Technol 6: 162167. Link: https://bit.ly/2Z1OTem

21. Ashraf S, Raza A, Aslam Z, Naeem H, Ahmed T (2020) Underwater Resurrection Routing Synergy using Astucious Energy Pods. J Robot Control JRC 1. Link: https://bit.ly/3eouwi7

22. Ashraf S, Ahmad A, Yahya A, Ahmed T (2020) Underwater Routing Protocols: Analysis of Link Selection Challenges. AIMS Electron Electr Eng 4: 200-215. Link: https://bit.ly/2VBwvbt

23. Kak N, Gupta R, Mahajan S (2010) Iris Recognition System. Int J Adv Comput Sci Appl 1. Link: https://bit.ly/2AP09D5

24. Sarfraz N (2019) Adermatoglyphia: Barriers to Biometric Identification and the Need for a Standardized Alternative. Cureus 11: e4040. Link: https://bit.ly/3hT8HsY

25. Kushwaha R, Nain N, Singal G (2017) Detailed Analysis of Footprint Geometry for Person Identification. In $201713^{\text {th }}$ International Conference on Signal-Image Technology Internet-Based Systems (SITIS). 229-236. Link: https://bit.ly/37NBCKC

26. Ashraf S, Saleem S, Ahmed T (2019) Sagacious Communication Link Selection Mechanism for Underwater Wireless Sensors Network. Int J Wirel Microw Technol 10: 12-25.

27. Hoo SC, Ibrahim H (2019) Biometric-Based Attendance Tracking System for Education Sectors: A Literature Survey on Hardware Requirements. 2019: 1-25. Link: https://bit.ly/2Z3ZpSG

28. Ashraf S, Ahmed T (2016) Machine Learning Shrewd Approach For An Imbalanced Dataset Conversion Samples. J Engneering Technol 10: 7-25.

29. Jain $Y$, Juneja M, Student M (2018) Ridge Energy Based Human Verification Using Iris and Palm Print. 5: 7. Link: https://bit.ly/2Z3ZLbY

30. ECIC. Link: https://bit.ly/316X0Oq

31. Ashraf S (2020) Underwater Routing Protocols Analysis of Intrepid Link Selection Mechanism, Challenges and Strategies. Int J Sci Res Comput Sci Eng 8: 1-9. Link: https://bit.ly/2NiUUOt

32. What Are Log-Gabor Filters and Why Are They Good?. Link: https://bit.ly/3dl87kl

33. Kapoor P, Lee D, Kim B, Lee S (2020) Computation-Efficient Quantization Method for Deep Neural Networks. Link: https://bit.ly/2NhQWp9

34. Ashraf S, Ahmed T, Raza A, Naeem H (2020) Design of Shrewd Underwater Routing Synergy Using Porous Energy Shells. Smart Cities 3: 74-92. Link: 
https://bit.ly/3hTCphx

35. Ashraf S, Muhammad D, Khan MA, Ahmed T (2019) Fuzzy based efficient Cosmetology Paradigm. Int J Multidiscip Curr Res 8: 421-425.

36. Ashraf S, Gao M, Mingchen Z, Ahmed T, Raza A, et al. (2020) USPF: Underwater Shrewd Packet Flooding Mechanism through Surrogate Holding Time. Wirel Commun Mob Comput 2020: 12. Link: https://bit.ly/3hT9sIM
37. What is false rejection rate (FRR)? Webopedia Definition. Link: https://bit.ly/3fL2JZj

38. Ashraf S, Ahmed T, Saleem S, Aslam Z (2020) Diverging Mysterious in Green Supply Chain Management. Orient J Comput Sci Technol 13: 22-28. Link: https://bit.ly/2B1xFGb

39. Ashraf S, Yahya A, Khan MA Culminate coverage for sensor network through Bodacious-instance Mechanism. Manag. J Wirel Commun Netw 8: 1-7.
Discover a bigger Impact and Visibility of your article publication with

Peertechz Publications

\section{Highlights}

* Signatory publisher of ORCID

* Signatory Publisher of DORA (San Francisco Declaration on Research Assessment)

* Articles archived in worlds' renowned service providers such as Portico, CNKI, AGRIS, TDNet, Base (Bielefeld University Library), CrossRef, Scilit, J-Gate etc.

* Journals indexed in ICMJE, SHERPA/ROMEO, Google Scholar etc.

- OAI-PMH (Open Archives Initiative Protocol for Metadata Harvesting)

* Dedicated Editorial Board for every journa

- Accurate and rapid peer-review process

* Increased citations of published articles through promotions

* Reduced timeline for article publication

Submit your articles and experience a new surge in publication services (https://www.peertechz.com/submission).

Peertechz journals wishes everlasting success in your every endeavours.

Copyright: (c) 2020 Ashraf S, et al. This is an open-access article distributed under the terms of the Creative Commons Attribution License, which permits unrestricted use, distribution, and reproduction in any medium, provided the original author and source are credited. 